\title{
Regional difference in optimal determinants of acute pulmonary vein reconnection following pulmonary vein isolation with high-power, short-duration radiofrequency exposure in patients with paroxysmal atrial fibrillation
}

\author{
Kyoichiro Yazaki ${ }^{1}$, Koichiro Ejima ${ }^{1}$, Miwa Kanai ${ }^{2}$, Shohei Kataoka ${ }^{2}$, SATOSHI \\ HIGUCHI $^{1}$, Daigo Yagishita ${ }^{3}$, Shoda Morio ${ }^{1}$, and Nobuhisa Hagiwara ${ }^{1}$
}

${ }^{1}$ Tokyo Women's Medical University

${ }^{2}$ Tokyo Women's Medical University Hospital

${ }^{3}$ Tokyo Women's Medical University

May 26, 2020

\begin{abstract}
Introduction: Acute pulmonary vein reconnection (PVR) is associated with longer procedure time and radiofrequency time during pulmonary vein isolation (PVI). However, determinants of acute PVR after high-power, short-duration PVI (HPSDPVI) in the guidance with unipolar signal modification (USM) remain unclear. Methods and Results: We evaluated 62 patients (age, 62 \pm 12 y; 45 men) with paroxysmal atrial fibrillation undergoing USM-guided HPSD-PVI. A 50-W radiofrequency (RF) was applied for 3-5 s after unipolar signal modification. In the segments adjacent to the esophagus (SAE), RF time was limited to $5 \mathrm{~s}$. Each circle was subdivided into 12 segments. For each radiofrequency tag within the circle, possible predictors of acute PVR, including minimum contact force, minimum force-time integral, minimum ablation index (AImin), minimum impedance drop (Imp-min), and maximum inter-lesion distance (ILDmax) were assessed. Acute PVR was observed in 43 (7\%) SAE and 21 $(17 \%)$ other segments $(\mathrm{p}=0.001)$. RF energy, RF application time and bilateral isolation time were $28 \pm 8 \mathrm{~kJ}, 10 \pm 3 \mathrm{~min}$, and $27 \pm 11 \mathrm{~min}$, respectively. Imp-min and ILDmax had the highest area under the curve (0.69 and 0.68) and of all indices, and were the sole independent predictors of acute PVR in segments other than the SAE and SAE, respectively, after adjusting for other cofounders (odds ratio [OR]: 0.90 [0.85-0.95], $\mathrm{p}=0.0003$; and OR: 1.39 [1.11-1.74], $\mathrm{p}=0.005$ ). Conclusions: In HPSD-PVI, a non-negligible amount of acute PVR was still observed, which was possibly dealt with an optimal target value of impedance drop and lesion distance.
\end{abstract}

\section{Methods}

Sixty-two participants who had undergone RF catheter ablation (RFA) for paroxysmal AF (PAF) using HPSD-PVI strategy from October 2018 to June 2019 were evaluated. They were subjected to circumferential PVI without any substrate modifications. 3-D cardiac computed tomography (CT) with or without contrast agent and transthoracic echocardiography were performed before the procedure. Transesophageal echocardiography was performed for patients with a high CHADS2 score ([?]2 points) to rule out thrombus in the left atrium. All antiarrhythmic drugs were stopped for at least five half-lives before the procedure. The study was approved by our institutional review board (Tokyo Women's Medical University) and was performed according to the institutional guidelines. All patients provided written informed consent.

\section{Catheter ablation protocol}

The details of the catheter ablation protocol have been previously published ${ }^{[6]}$. Under uninterrupted anti- 
coagulants, all patients underwent PVI and superior vena cava isolation (SVCI). These were performed with geometric information based on reconstructed 3-D CT imaging system (CARTO 3, Biosense Webster, Inc., Diamond Bar, CA). Two long sheaths with one circular multi-electrode catheter and a 3.5-mm open-irrigated tipped catheter (ThermoCool STSF; Biosense Webster Inc., Diamond Bar, CA, USA) were used as ablation tools. To monitor the esophageal temperature, a multi-electrode esophageal temperature-monitoring probe was employed during the procedure (Esophaster, Japan Lifeline, Tokyo, Japan).

The detail of radiofrequency power was $50 \mathrm{~W}$ with an upper limit temperature of $42^{\circ} \mathrm{C}$ as recently denoted. ${ }^{[5]}$ Disappearance of negative deflection in the unipolar electrogram recorded at the distal tip of the ablation catheter (unipolar signal modification, USM; Figure 1) was adopted as an indicator of sufficient transmural necrosis. ${ }^{[4,7]}$ RFA was continued for 3-5 seconds after the USM in the segments other than those adjacent to the esophagus (SAE). RF time was limited to $5 \mathrm{~s}$ or by the alert of the temperature sensor (upper limit of $39^{\circ} \mathrm{C}$ ) to avoid esophageal injury in the SAE. During each RF application, the modification of the unipolar atrial electrogram was monitored in real-time at a sweep speed of $200 \mathrm{~mm} / \mathrm{s}$ and filtered with a $0.5-120 \mathrm{~Hz}$ by the CARTO system.

To clearly visualize unipolar signals, a 10-polar electrode catheter with an indifferent electrode (DECANAV ${ }^{\circledR}$, Biosense Webster Inc., Diamond Bar, CA, USA) in the coronary sinus was used, where a reference annotation signal was recorded from the bipolar signals. Targeted lesion distance was $<5 \mathrm{~mm}$ and contact force $(\mathrm{CF})$ was aimed at $5-20 \mathrm{~g}$ (target $10 \mathrm{~g}$ ) and at $<10 \mathrm{~g}$ in the segments other than SAE and in the SAE, respectively. RFA was stopped at $3 \mathrm{~s}$ after the USM in case CF was above $15 \mathrm{~g}$ or catheter was perpendicularly placed on the atrial wall. RFA was continued for $5 \mathrm{~s}$ after the USM if CF was below $10 \mathrm{~g}$ or RFA site was near the carina. Intensive induction of atrial overdrive pacing with isoproterenol infusion and the confirmation of the absence of dormant conduction with adenosine-triphosphate (ATP) infusion was attempted. The confirmation was conducted for at least $20 \mathrm{~min}$ after the isolation of the ipsilateral PV pair.

\section{Regional assessment of acute PVR}

Acute PVR was defined as spontaneous or isoproterenol-induced PVR, or adenosine-induced dormant conduction. Each left atrial (LA) antrum was divided into 6 regions, including supero-/infero-anterior, supero/infero-posterior, roof, and bottom, and resulted in 12 segments. Here, relevant parameters were analyzed, and the minimum values were identified for each segment such as minimum contact force (CFmin), minimum force-time integral (FTImin), minimum ablation index (AImin), maximum inter-lesion distance (ILDmax), and minimum impedance drop (Imp-min). Changes in three-dimensional-mapping-related indices were visualized using an on-site monitor. Total impedance decrease measured at every $100 \mathrm{~ms}$ was monitored in graph viewer and later exported for processing, considering fluctuation related to respiratory cycle (Figure 1).

\section{Follow-up after the procedure}

All patients were given follow-up in the outpatient clinic at 1,3,6, 9, and 12 months after the procedure and every 6 months thereafter. Atrial tachyarrhythmia (ATA) recurrence was evaluated according to patient symptoms, electrocardiographic recordings, and 24-h ambulatory monitoring (3, 6, 9, and 12 months after the ablation and every 6 months thereafter). Patients with palpitations were encouraged to use portable electrocardiographic monitoring (HCG-801R; Omron, Kyoto, Japan). Recurrence was defined as recurrent symptoms and/or detection of ATAs using aforementioned modalities or data provided by cardiac implantable electrical devices (lasting $>30 \mathrm{~s}$ ) after a 2-month blanking period from the ablation procedure without any anti-arrhythmic drugs (AADs). If AAD was administered for patient after the procedure, it was considered as "ATA recurrence."

\section{Statistical analysis}

Categorical variables were expressed as the mean \pm standard deviation (SD) or median with interquartile range (IQR). Student's t-test was used to compare characteristics of the segments with acute PVR and those without. Receiver-operating characteristics (ROC) curve analysis was utilized to identify the most optimal 
cut-off value (with a Youden index) to predict acute PVR among various indices, and to determine the cut-off value with the specificity of $90 \%$. Logistic regression analysis was performed to evaluate predictors of acute PVR in univariate and multivariate model for all segments, and region-specific analysis. The log-rank test was carried out for Kaplan-Meier curve analysis to assess cumulative rate of ATA-free survival in all subjects. All tests were considered statistically significant at $P$-value $<0.05$. All statistical analyses were performed with $\mathrm{JMP}^{\circledR} 13$ (SAS Institute Inc., Cary, NC, USA).

\section{Results}

\section{Background characteristics and clinical outcome}

The baseline and procedural characteristics in 62 patients were given in Table 1. Structural heart disease (SHD) was found in 11 (15\%) patients, and median AF history of 8 months was noted. An average procedure time was 125 minutes with RF time and energy for PVI of $10 \pm 3$ min and $28 \pm 8 \mathrm{~kJ}$, respectively. All patients had undergone superior vena cava isolation after PVI. Complication occurred in only one patient: an acute right phrenic nerve injury was identified following completion of the right-sided PVI before superior vena cava isolation. This was almost fully recovered after 6-month follow up.

\section{Region-specific analysis of acute PVR}

In a total of 620 segments other than SAE and 124 SAE, acute PVR was observed in 43 (7\%) and 21 (17\%) segments $(\mathrm{p}=0.001)$, respectively. The detailed distribution of acute PVR sites is depicted in the Figure 2 . In SAE, there was only a significant difference in ILDmax between the segments with acute PVR and those without; whereas in the segments other than SAE, there was a significant difference in CFmin, FTImin, AImin, and Imp-min (Table 2). In the ROC curve analysis, ILDmax and Imp-min had the highest area under the curve (AUC) to predict the absence of acute PVR in the SAE and the segments other than SAE, respectively (0.68 and 0.69 ) among various 3 -D mapping related indices.

ILDmax of $4.8 \mathrm{~mm}$ discriminated durable segments with a specificity of $90 \%$ in the SAEs and also in the entire segments. Conversely, an Imp-min of $6.5 \mathrm{ohm}$ could distinguish segments with gaps and no gaps with a specificity of $90 \%$ in segments other than SAE. In entire-segment analysis, AI min of 383 au was required to discern durable lesion with a specificity of $90 \%$, while AI of 405 au for the segments other than SAE and AI of 334 au for the SAE were related to the absence of acute PVR with a specificity of $90 \%$.

\section{Predictors of acute PVR}

Table 3 shows the odds ratio of acute PVR in various 3-D mapping-related indices. Multivariate analysis indicated that ILDmax and Imp-min were sole independent predictors of acute PVR in the SAE and segments other than SAE, respectively.

\section{Follow-up and late outcome}

With the median follow-up of 12.8 months, ATA-free rate was $81 \%$ and $72 \%$ at 6 months and 1 year after the procedure, respectively. Excluding patients with SHD increased this value to $86 \%$ and $83 \%$ at 6 months and 1 year after the procedure, respectively. Late adverse event was not observed during the follow-up period. Of patients with late recurrence, 12 patients underwent a redo procedure. Ten patients had the segments with late PVRs which consisted of 1 left infero-anterior, 4 left infero-posterior, 5 left supero-posterior, 3 right infero-anterior, 1 right bottom, 1 right infero-posterior, 2 right supero-anterior, and 5 right supero-posterior: $9(38 \%)$ SAEs versus $17(14 \%)$ segments other than SAE ( $\mathrm{p}=0.01)$. Only three segments with late PVR matched those with acute ones. There was no significant difference in 3-D mapping-related indices between the segments with late PVR and those without. Only Imp-min tended to be higher in the segments other than SAE without late PVRs than in those with $(4.7 \pm 4.3$ versus $2.7 \pm 3.3, \mathrm{p}=0.09)$.

\section{Discussion}

Main findingsIn patients with PAF, HPSD-PVI achieved relatively short procedure and isolation time, and acceptable late outcome in the guidance with USM. Here, ILDmax and Imp-min independently predicted 
acute PVR during procedure in the SAE and the segments other than SAE, respectively, after adjusting for potential cofounders.

\section{Validity and safety of HP strategy}

Recent clinical studies revealed the efficacy and safety of HPSD-PVI over conventional PVI at normal power and duration, ${ }^{[8-10]}$ where RF time and energy could be reduced. ${ }^{[2,3]}$ The result shown here concurred with that of previous reports with total RF time of 10 min and RF energy of $29 \mathrm{~kJ}$ for PVI. ${ }^{[2,11]}$

Theoretically, HPSD-PVI could lessen collateral damage with a shallow and wide lesion creation due to resistive heating. ${ }^{[12,13]}$ Clinically, Winkle et al have recently demonstrated the extremely low complication rate using HPSD-PVI, where a smaller number of atrio-esophageal fistula was observed compared to the conventional strategy. ${ }^{[14]}$ Similarly, by assessing esophageal thermal injury after HPSD-PVI by late gadolinium enhancement magnetic resonance imaging within 24 hours post-procedure, Baher et al concluded that the distribution and severity of esophageal damage was similar between HPSD-PVI and low power long duration groups. ${ }^{[15]}$ These data highlighted the safeness of HPSD-PVI over conventional strategy. In the present study, only 1 patient with an enlarged LA had experienced acute right phrenic nerve injury after right-sided PVI, although this partially recovered within half a year. Subsequently, a 10-mA stimulation test was routinely performed at the right superior PV antrum to avoid phrenic nerve injury. In patients with breathing disorder, deep sedation could hinder HPSD-PVI due to unstable catheter contact. Therefore, moderate anesthesia with dexmedetomidine could keep optimal condition without evoking breathing disorder in such cases. Similarly, controlled tidal volume using mechanical ventilator could allow for HPSD-PVI even in patients under general anesthesia.

\section{Indication for lesion creation in HP strategy}

Several attempts have been conducted to increase ATA-free survival rate, including various guidance during PVI (CF, FTI, AI, or impedance drop), or confirmation of acute PVR using isoproterenol infusion or ATP after PVI, which was considered responsible for durable lesion creation. Most importantly, however, all of acute PVRs were eliminated by touch-up procedure in the same session, and there had been enough waiting time (20 min) after the final ipsilateral PVI, which rendered the lesion creation robust. In these respects, we could not entirely expect additional effect of HPSD-PVI on the late outcome as previously described; $[1,2,16]$ however, further examination of acute PVR is required due to its substantial association with longer RF time and larger RF energy delivery, which in turn leads to overheating of tissue. Thus, optimal measurements for the durable lesion creation is needed for HPSD-PVI.

Although AI was a reliable indicator for lesion creation in PVI at normal power and duration, whether it was also suitable in HPSD-PVI remains uncertain. Some researchers reported the efficacy of HPSD-PVI guided by AI value without any in-procedure major complications. ${ }^{[1,11]}$ In recent literature, the same finding was noted in lesion size index (LSI)-guided HPSD-PVI [17]. However, neither AI nor LSI could directly exacerbate tissue damage; suggesting these were device-related parameters. Furthermore, since CF depends on the contact sensor at the distal tip of the ablation catheter, sensor error occurrence is relatively common. Indeed, while with specificity of $90 \%$ AI of 405 and 334 au for the segments other than SAE and SAE, respectively, were associated with durable lesion creation in the present study, such values were remarkably low compared with that applied in previous studies. ${ }^{[1,11]}$ Similarly, discrepancy between AI and impedance drop in the gap site were also observed, which could be explained by lower reliability of AI value. Figure 3 showed the comparison of VisTags of AI value and impedance drop, the latter of which clearly demonstrated gap site whereas the former could not. Interestingly, cases of low AI value but sufficient impedance drop after the RF application had been observed and resulted in absence of gaps. Figure 4 showed the relevant parameters in a durable site where sufficient impedance drop and moderate AI value was found. These examples suggest AI value to be a less reliable measurement than impedance drop. Since existing AI or LSI-guided HP strategy could deliver excessive RF energy to tissues, impedance drop might be a more sophisticated indicator of lesion creation, especially in the HP strategy.

In the present study of HPSD-PVI guided by USM, impedance drop solely and significantly predicted acute 
PVR after adjusting for AI and ILD. USM could directly enhance cellular necrosis without any collateral damages and impedance drop appeared to behave similarly both experimentally and clinically. ${ }^{[7], ~}{ }^{[18,19]}$ Figure 1 showed an example of a durable site in the procedure, demonstrating that R-wave pattern in unipolar signal was immediately $(5 \mathrm{~s})$ achieved after the RFA and reached a plateau; however, generator impedance gradually decreased, finally resulting in an absolute change of $13 \mathrm{ohm} 9 \mathrm{~s}$ after the RFA. This example could demonstrate the importance of continuous application after the achievement of USM to create durable lesion.

\section{Clinical implications of impedance-guided procedure}

As our strategy was not dependent on the AI value, exact AImin value for durable lesion creation was not determined. Although several data highlighted the significance of impedance drop in durable lesion creation, ${ }^{[18,20]}$ certain limitations need to be considered such as insufficiency without stable catheter contact $^{[21]}$ and influence by contact angle. ${ }^{[22]}$ To avoid esophageal injury, we could not apply HP application for up to 5 seconds; as such, impedance drop was not a preferable parameter to predict acute PVRs in the SAE. Nevertheless, a strong association was identified between ILDmax and acute PVR in the SAE, and ILDmax of $4.8 \mathrm{~mm}$ had a strong association with durability with a specificity of $90 \%$, suggesting that current protocol could be improved with tightened lesion creation. As Imp-min of $6.5 \mathrm{ohm}$ was strongly associated with an acute durability with a specificity of $90 \%$ in the segments other than SAE, impedance-guided HPSD-PVI with a target value of above $6.5 \mathrm{ohm}$ would be suitable in the future. Occasionally, unipolar signal could not be visible due to electrical artifacts even with an indifferent electrode; and unipolar signal could not be reflected by VisiTag on its nature. As impedance drop could be monitored on-site, and dynamic change visualized by VisiTag color, it could be used during PVI in persistent AF patients. While efficacy of impedance-guided HPSD-PVI requires further evaluation, providing sufficient catheter contacts and avoiding perpendicular contact, this technique might prove applicable.

\section{Study limitations}

Firstly, although the relatively small number of subjects might underpower the statistical analysis, in this persegments analysis, 768 regions were considered, which in turn could improve statistical confidence. Secondly, Imp-min and late recurrence had an associative tendency, although not significant in the segments other than SAE, which might be derived from a small number of subjects for statistics. Thirdly, acute PVRs were distributed heterogeneously; therefore, it was preferable to assess each segment to clarify the regional difference in the characteristics of acute PVRs, which requires more number of subjects for evaluation. Lastly, if the impedance-guided HPSD procedure is warranted for clinical practice, its safety aspects, especially regarding esophageal injury, should be assessed as they have not been sufficiently evaluated to date.

\section{Conclusions}

From our results, lesion distance and impedance drop determined the acute PVR in the SAE and the segments other than SAE, respectively, in USM-guided HPSD-PVI. This strategy could be improved with the guidance of impedance drop and lesion distance, allowing for reduction in total amount of RF time and energy as compared with existing HPSD strategy while maintaining an acceptable late outcome.

Acknowledgments: We would like to thank Editage (www.editage.jp) for English language editing.

\section{References}

1. Berte B, Hilfiker G, Russi I, et al. Pulmonary vein isolation using a higher power shorter duration CLOSE protocol with a surround flow ablation catheter. J Cardiovasc Electrophysiol 2019; 30(11):2199-2204.

2. Pambrun T, Durand C, Constantin M, et al. High-Power (40-50 W) Radiofrequency Ablation Guided by Unipolar Signal Modification for Pulmonary Vein Isolation. Circ Arrhythm Electrophysiol2019; 12 (6): p. e007304.

3. Kottmaier M, Poppa M, Bourier F, et al. Safety and outcome of very high-power short-duration ablation using $70 \mathrm{~W}$ for pulmonary vein isolation in patients with paroxysmal atrial fibrillation. Europace 2020;22(3):388-393. 
4. Ejima K, Henmi R, Iwanami Y, et al. Comparison of the Efficacy of Empiric Thoracic Vein Isolation for the Treatment of Paroxysmal and Persistent Atrial Fibrillation in Patients Without Structural Heart Disease. J Cardiovasc Electrophysiol 2017;28(3): p. 266-272.

5. Koichiro Ejima SH, Kyoichiro Yazaki, Shohei Kataoka, et al. Comparison of high-power and conventionalpower radiofrequency energy deliveries in pulmonary vein isolation using unipolar signal modification as a local endpoint. J Cardiovasc Electrophysiol2020. In press .

6. Bortone A, Brault-Noble G, Appetiti A, Marijon E Elimination of the negative component of the unipolar atrial electrogram as an in vivo marker of transmural lesion creation: acute study in canines. Circ Arrhythm Electrophysiol 2015; 8(4): p. 905-911.

7. Bortone A, Appetiti A, Bouzeman A, et al. Unipolar signal modification as a guide for lesion creation during radiofrequency application in the left atrium: prospective study in humans in the setting of paroxysmal atrial fibrillation catheter ablation.Circ Arrhythm Electrophysiol 2013. 6 (6): p. 1095-1102.

8. Winkle RA, Mead RH, Engel G, Patrawala RA. Atrial fibrillation ablation: "perpetual motion" of open irrigated tip catheters at $50 \mathrm{~W}$ is safe and improves outcomes. Pacing Clin Electrophysiol2011; 34 (5): p. 531-539.

9. Winkle RA, Moskovitz R, Mead RH, et al. Atrial fibrillation ablation using very short duration $50 \mathrm{~W}$ ablations and contact force sensing catheters. J Interv Card Electrophysiol 2018; 52(1): p. 1-8.

10. Dhillon G, Ahsan S, Honarbakhsh S, et al. A multicentered evaluation of ablation at higher power guided by ablation index: Establishing ablation targets for pulmonary vein isolation. J Cardiovasc Electrophysiol 2019; 30(3):357-365.

11. Chen S, Schmidt B, Bordignon S, et al. Ablation index-guided $50 \mathrm{~W}$ ablation for pulmonary vein isolation in patients with atrial fibrillation: Procedural data, lesion analysis, and initial results from the FAFA AI High Power Study. J Cardiovasc Electrophysiol2019. 30(12): p. 2724-2731.

12. Bhaskaran A, Chik W, Pouliopoulos J, et al. Five seconds of 50-60 W radio frequency atrial ablations were transmural and safe: an in vitro mechanistic assessment and force-controlled in vivo validation. Europace 2017;19(5): p. 874-880.

13. Bourier F, Duchateau J, Vlachos K, et al. High-power short-duration versus standard radiofrequency ablation: Insights on lesion metrics. J Cardiovasc Electrophysiol 2018; 29(11): p. 1570-1575.

14. Winkle RA, Mohanty S, Patrawala RA, et al. Low complication rates using high power (45-50 W) for short duration for atrial fibrillation ablations. Heart Rhythm 2019;16(2): p. 165-169.

15. Baher A, Kheirkhahan M, Rechenmacher SJ, et al. High-Power Radiofrequency Catheter Ablation of Atrial Fibrillation: Using Late Gadolinium Enhancement Magnetic Resonance Imaging as a Novel Index of Esophageal Injury. JACC: Clin Electrophysiol 2018;4(12): p. 1583-1594.

16. Bunch TJ, May HT, Bair TL, et al. Long-term outcomes after low power, slower movement versus high power, faster movement irrigated-tip catheter ablation for atrial fibrillation. Heart Rhythm 2020; 17(2):184189

17. Castrejón-Castrejón S, Martínez Cossiani M, Ortega Molina M, et al. Feasibility and safety of pulmonary vein isolation by high-power short-duration radiofrequency application: short-term results of the POWERFAST PILOT study. J Interv Cardiovasc Electrophysiol2020; 57(1):57-65.

18. Chinitz JS, Kapur S, Barbhaiya C, et al. Sites With Small Impedance Decrease During Catheter Ablation for Atrial Fibrillation Are Associated With Recovery of Pulmonary Vein Conduction. J Cardiovasc Electrophysiol 2016; 27(12): p. 1390-1398.

19. Inaba O, Nagata Y, Sekigawa Masahiro, et al. Impact of impedance decrease during radiofrequency current application for atrial fibrillation ablation on myocardial lesion and gap formation.J Arrhythm 2018; 
34(3): p. 247-253.

20. Ikeda A, Nakagawa H, Lambert H, et al. Relationship between catheter contact force and radiofrequency lesion size and incidence of steam pop in the beating canine heart: electrogram amplitude, impedance, and electrode temperature are poor predictors of electrode-tissue contact force and lesion size. Circ Arrhythm Electrophysiol 2014;7 (6): p. 1174-80.

21. Reichlin T, Knecht S, Lane C, et al. Initial impedance decrease as an indicator of good catheter contact: insights from radiofrequency ablation with force sensing catheters. Heart Rhythm 2014; 11(2): p. 194-201.

22. Knecht S, Reichlin T, Pavlovic N, et al. Contact force and impedance decrease during ablation depends on catheter location and orientation: insights from pulmonary vein isolation using a contact force-sensing catheter. J Interv Card Electrophysiol 2015; 43(3): p. 297-306.

23. Anter E, Tschabrunn CM, Contreras-Valdes FM, Buxton AE, Josephson ME. Radiofrequency ablation annotation algorithm reduces the incidence of linear gaps and reconnection after pulmonary vein isolation. Heart Rhythm 2014; 11(5): p. 783-790.

Figure legends

\section{Figure 1. Unipolar signal modification and impedance changes}

Example of a change in unipolar electrogram with dynamic changes in generator impedance. S-wave of unipolar signal disappeared three seconds after the radiofrequency application (RFA) according to significant impedance decrease, and R-pattern was achieved five seconds after the RFA. After that, impedance gradually decreased from $133 \mathrm{ohm}$, and finally reached $120 \mathrm{ohm}$ while a notch in positive unipolar electrogram completely disappeared.

\section{Figure 2. The distribution of acute pulmonary vein reconnection site}

The site of acute pulmonary vein reconnection (PVR) including time-dependent PVR and adenosine-induced PVR was indicated by colored circles; as annotated in the figure.

ATP, adenosine triphosphate; RPV, right pulmonary vein; LPV, left pulmonary vein

\section{Figure 3. The example of relevant parameters in a gap site}

In a posterior-anterior projection, VisiTags indicated ablation index (A) and impedance drop (B) of each lesion. The gap site was indicated by yellow circle at the right supero-posterior segment. Graph viewer demonstrated dynamic change of impedance and contact force by green and blue, respectively. Fluctuation of impedance was observed (C). Panel (D) showed the parameters corresponding to the gap site. Eso, esophagus

Figure 4. The example of relevant parameters in a durable site

In a posterior-anterior projection, VisiTags indicated the ablation index (A) and impedance drop (B) of each lesion. The durable site was indicated by a yellow circle at the supero-anterior segment. Graph viewer demonstrated dynamic change of impedance and contact force by green and blue, respectively. Gradual and stable decrease of impedance was observed (C). Panel (D) showed the parameters corresponding to the durable site. Eso, esophagus

\section{Tables}

Table 1. Baseline and Characteristics

\begin{tabular}{llll}
\hline Baseline and Characteristics & Baseline and Characteristics & Baseline and Characteristics & Baseline and Characte \\
Mean age, years & Mean age, years & Mean age, years & Mean age, years \\
Male, n (\%) & Male, n (\%) & Male, n (\%) & Male, n (\%) \\
Structural heart disease, n (\%) & Structural heart disease, n (\%) & Structural heart disease, n (\%) & Structural heart disea
\end{tabular}


Echocardiographic parameter

History of AF, months

Location of esophagus

Left/Right/Middle, n

Left common pulmonary vein

Right middle pulmonary vein

RF time for PVI, min

RF energy for PVI, kJ

Time for bilateral PVI, min

Time for the left PVI, min

Time for the right PVI, min

Total procedure time, min

Bilateral isolation length, mm

Radiation exposure, min

Additional ablation
Echocardiographic parameter

History of AF, months

Location of esophagus

Left/Right/Middle, n

Left common pulmonary vein

Right middle pulmonary vein

RF time for PVI, min

RF energy for PVI, kJ

Time for bilateral PVI, min

Time for the left PVI, min

Time for the right PVI, min

Total procedure time, min

Bilateral isolation length, mm

Radiation exposure, min

Additional ablation
Echocardiographic parameter

LAVI, $\mathrm{ml} / \mathrm{cm} 2$

LVEF, \%

History of AF, months

Location of esophagus

Left/Right/Middle, n

Left common pulmonary vein

Right middle pulmonary vein

RF time for PVI, min

RF energy for PVI, $k J$

Time for bilateral PVI, min

Time for the left PVI, min

Time for the right PVI, min

Total procedure time, min

Bilateral isolation length, mm

Radiation exposure, min

Additional ablation

SVC isolation, $\mathrm{n}(\%)$

CTI linear ablation, n (\%)

AT ablation, n (\%)

Non-PV foci ablation, n (\%)
Echocardiographic pas

History of AF, month Location of esophagus Left/Right/ Left common pulmon Right middle pulmonء RF time for PVI, min RF energy for PVI, $\mathrm{k}$. Time for bilateral PV Time for the left PVI, Time for the right PV Total procedure time, Bilateral isolation len Radiation exposure, $\mathrm{n}$ Additional ablation SVC isolation, $\mathrm{n}(\%)$ CTI linear ablation, $\mathrm{n}$ AT ablation, n (\%) Non-PV foci ablation,

Values are expressed as n (\%), mean \pm SD, and median [IQR]

LAVI, left atrial volume index; LVEF, left ventricular ejection fraction; AF, atrial fibrillation; PVI, pulmonary vein isolation; RF, radiofrequency; SVC, superior vena cava; CTI, cavo-tricuspid isthmus; AT, atrial tachycardia; PV, pulmonary vein

Table 2. The difference of 3-D mapping related indices in the segments with and without acute PVR

\begin{tabular}{lllllll}
\hline \multirow{2}{*}{ Variables } & Other than SAE & Other than SAE & Other than SAE & SAE & SAE & SAE \\
& PVR $(+)$ & PVR $(-)$ & P-value & PVR $(+)$ & PVR $(-)$ & P-value \\
CFmin, g & $\mathrm{N}=48$ & $\mathrm{~N}=602$ & & $\mathrm{~N}=21$ & $\mathrm{~N}=109$ & \\
FTImin, gs & $5.4 \pm 2.7$ & $7.4 \pm 3.0$ & 0.01 & $6.7 \pm 2.4$ & $7.8 \pm 3.5$ & 0.82 \\
AImin, au & $334 \pm 53$ & $67 \pm 35$ & 0.02 & $37 \pm 18$ & $43 \pm 27$ & 0.17 \\
ILDmax, mm & $6.7 \pm 1.8$ & $350 \pm 54$ & 0.02 & $287 \pm 35$ & $298 \pm 48$ & 0.20 \\
Imp-min, ohm & $3.2 \pm 4.1$ & $6.6 \pm 1.7$ & 0.69 & $6.9 \pm 1.9$ & $5.9 \pm 1.4$ & 0.001 \\
\hline
\end{tabular}

Values are expressed as mean \pm SD

SAE, segments adjacent to esophagus; PVR, pulmonary vein reconnection; CFmin, minimum contact force; FTImin, minimum force-time integral; AImin, minimum ablation index; ILDmax, maximum inter-lesion distance; Imp-min, minimum impedance drop

Table 3. The predictors of acute PVR

\begin{tabular}{clllll}
\hline & Univariate analysis & Univariate analysis & Univariate analysis & Univariate analysis & Univariate analys \\
& Other than SAE & Other than SAE & Other than SAE & SAE & SAE \\
Variables & OR & $95 \%$ CI & P value & OR & $95 \%$ CI \\
AImin, au & 0.99 & $0.99-1.00$ & 0.02 & 0.99 & $0.99-1.00$ \\
ILDmax, $\mathrm{mm}$ & 1.02 & $0.89-1.18$ & 0.69 & 1.42 & $1.13-1.78$
\end{tabular}


Imp-min, ohm

CFmin, g

FTImin, gs
0.89

0.88

0.99
$0.83-0.94$

$0.83-0.94$
$0.79-0.97$

$0.98-0.99$

$<0.0001$

0.01

0.01
0.95

0.89

0.99
$0.85-1.06$

$0.78-1.02$

$0.97-1.01$

\begin{tabular}{|c|c|c|c|c|c|}
\hline & $\begin{array}{l}\text { Multivariate analysis } \\
\text { Other than SAE }\end{array}$ & $\begin{array}{l}\text { Multivariate analysis } \\
\text { Other than SAE }\end{array}$ & $\begin{array}{l}\text { Multivariate analysis } \\
\text { Other than SAE }\end{array}$ & $\begin{array}{l}\text { Multivariate analysis } \\
\text { SAE }\end{array}$ & $\begin{array}{l}\text { Multivaric } \\
\text { SAE }\end{array}$ \\
\hline Variables & OR & $95 \% \mathrm{CI}$ & $\mathrm{P}$ value & OR & $95 \% \mathrm{CI}$ \\
\hline AImin, au & 0.99 & $0.99-1.00$ & 0.19 & 0.99 & $0.98-1.0$ \\
\hline ILDmax, mm & 1.01 & $0.88-1.17$ & 0.87 & 1.39 & $1.11-1.7$ \\
\hline Imp-min, ohm & 0.90 & $0.85-0.95$ & 0.0003 & 0.97 & $0.87-1.0$ \\
\hline
\end{tabular}

SAE, segments adjacent to esophagus; OR, odds ratio; CI, confidence interval; CFmin, minimum contact force; FTImin, minimum force-time integral; AImin, minimum ablation index; ILDmax, maximum interlesion distance; Imp-min, minimum impedance drop

Fig 1
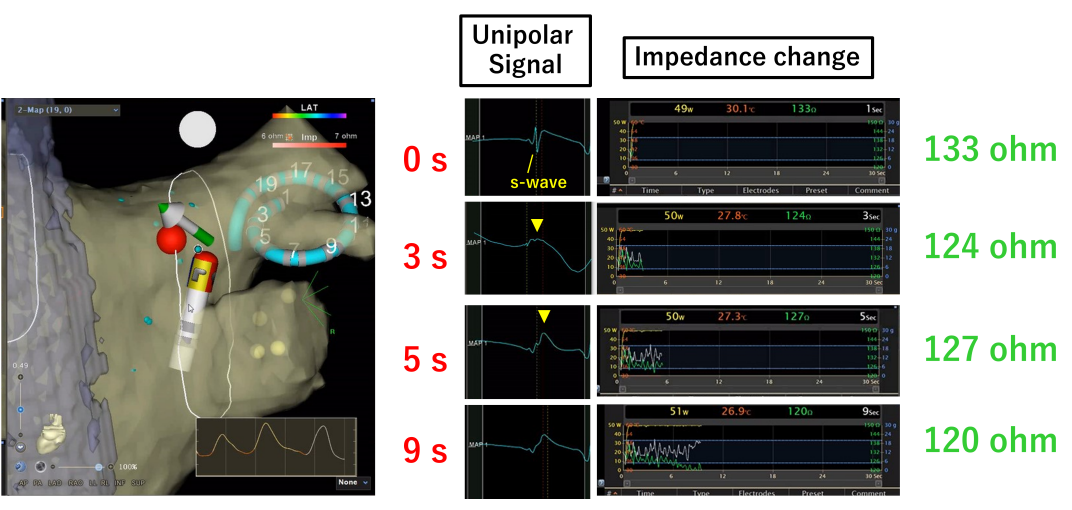

Fig 2
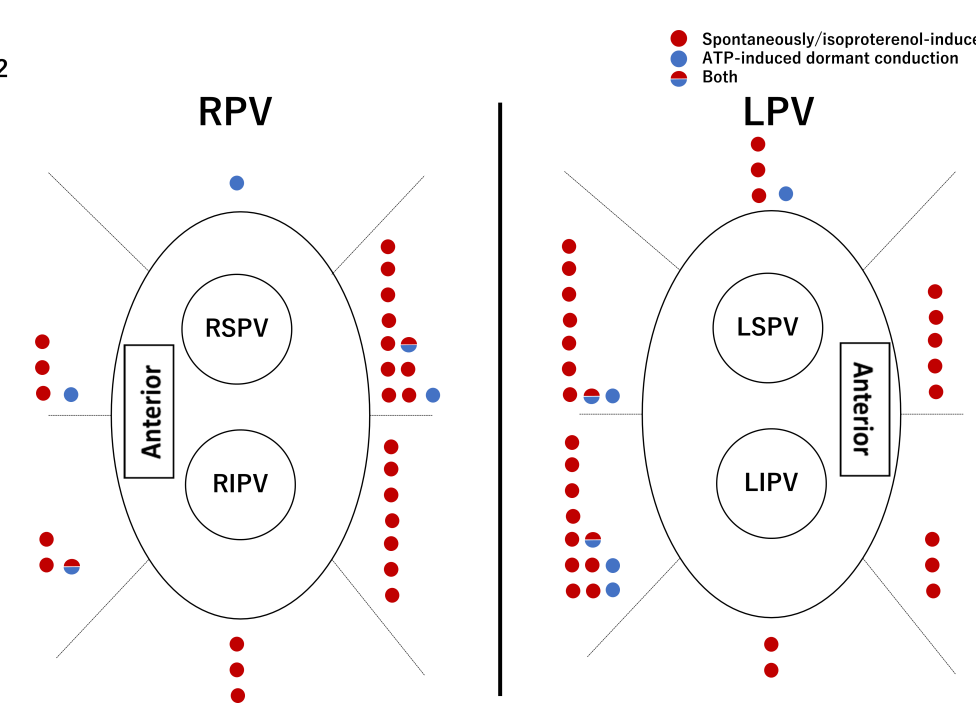
Fig 3
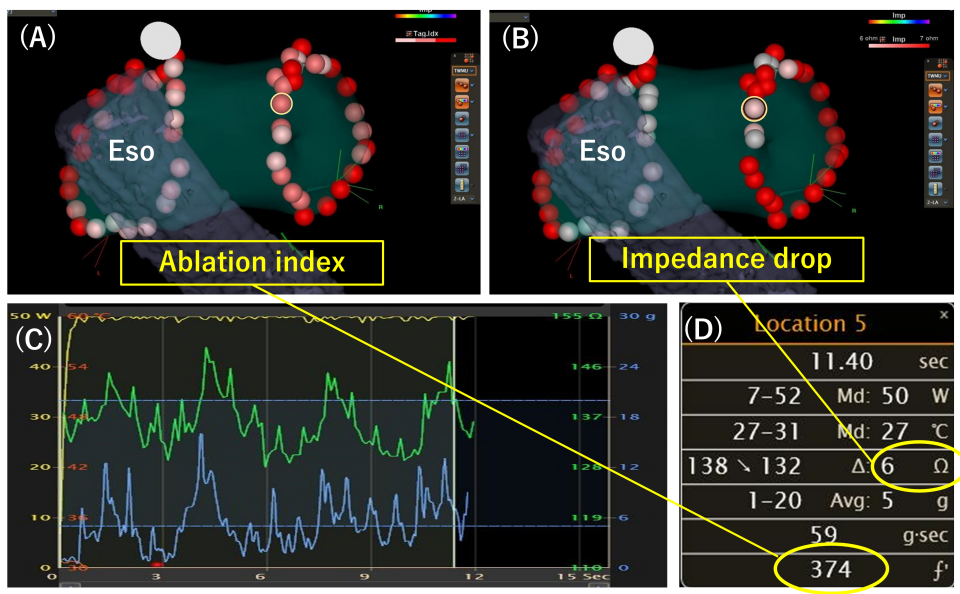

Fig 4
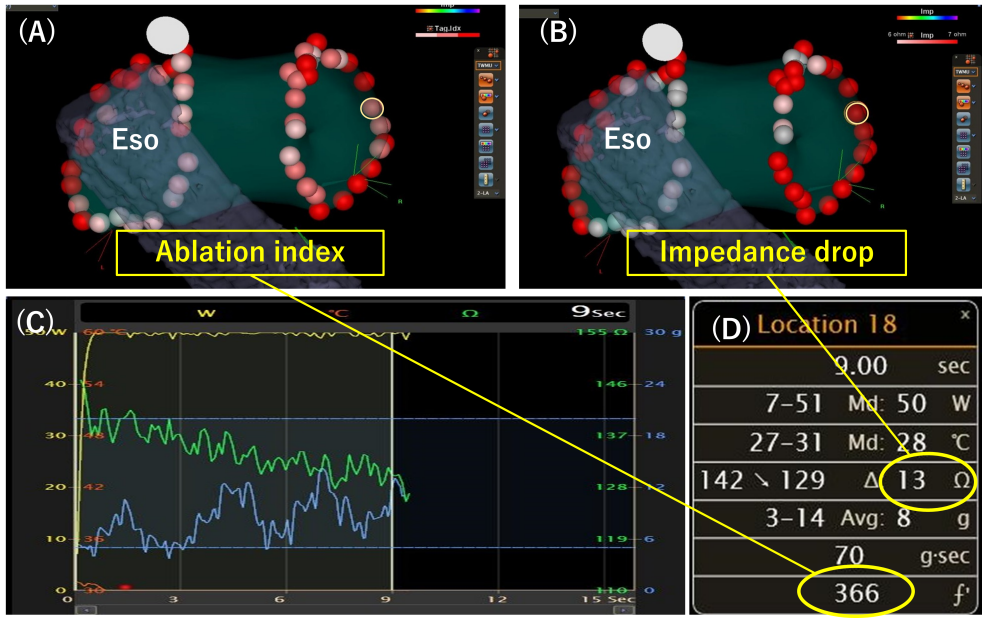

(D) Locdtion 18

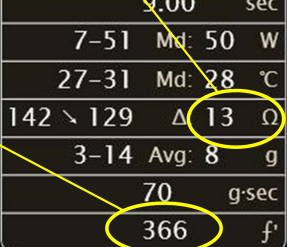

